\title{
EL CONTROL PRESIDENCIAL DE LA AGENDA LEGISLATIVA EN AMÉricA LATINA*
}

\author{
Presidential control of the legislative agenda in Latin America
}

\section{MANOEL LEONARDO SANTOS}

Universidade Federal de Minas Gerais

\section{ANÍBAL PÉREZ-LIÑÁN}

University of Pittsburgh

\section{MERCEDES GARCÍA MONTERO}

Universidad de Salamanca

\begin{abstract}
RESUMEN
Este trabajo analiza bajo qué condiciones los presidentes logran dominar la agenda legislativa. Para abordar esta cuestión analizamos los períodos gubernamentales de 30 presidentes latinoamericanos, desarrollando un análisis cualitativo comparativo (QCA). El análisis destaca que la combinación de un diseño institucional favorable y una división del Congreso en bloques ideológicos diferenciados favorece al poder ejecutivo. Otras dos condiciones, la existencia de una mayoría parlamentaria afín y la ausencia de fragmentación partidaria, se combinan con los elementos anteriores para garantizar el predominio del ejecutivo. Las conclusiones de este estudio comparativo son confirmadas por un análisis estadístico complementario.
\end{abstract}

Palabras clave: Poder legislativo; relaciones ejecutivo-legislativo; producción legislativa; América Latina; análisis cualitativo comparativo (QCA).

\begin{abstract}
This paper analyzes the conditions under which presidents dominate the legislative agenda. To address this issue we analyze the governmental period of 30 Latin American Presidents, developing a qualitative comparative analysis (QCA). The analysis highlights that the combination of a favorable institutional design and a division of Congress in different ideological blocs favors the Executive branch. Two other conditions, reliance on a parliamentary majority and the absence of party fragmentation, are combined with the above elements to ensure the predominance of the Executive. The findings of this comparative study are confirmed by a supplementary statistical analysis.
\end{abstract}

Key words: Legislative branch; Executive-Legislative relations; Legislative production; Latin America; Qualitative comparative analysis (QCA).

* $\quad$ Este artículo se enmarca dentro del proyecto de investigación, dirigido por Fátima García Díez: “Actividad legislativa, carrera política y sistema electoral: cómo se configura la representación política en América Latina"(CSO2012-39377-C02-02), financiado por el Ministerio de Economía y Competitividad de España. 
¿Qué factores garantizan al Poder Ejecutivo el control sobre la agenda legislativa de su país? Si bien la función legislativa se asocia naturalmente al parlamento, el Poder Ejecutivo desempeña un papel crucial como iniciador de propuestas legislativas en todas las democracias contemporáneas. La reflexión sobre esta realidad ha conducido a varios observadores a afirmar que los parlamentos han perdido centralidad en el proceso político para transformarse en meros ratificadores de las decisiones que toma el Ejecutivo. ${ }^{1}$ Estas críticas también han alcanzado a las democracias latinoamericanas, en tanto algunos autores estiman que un elemento característico de la región es la existencia de presidentes con gran influencia y de parlamentos débiles (Nino, 1996; O'Donnell, 1994).

Sin embargo, solo algunos presidentes logran monopolizar la iniciativa legislativa. Entre las razones esgrimidas para explicar el predominio de ciertos presidentes en América Latina se han mencionado los cuantiosos poderes legislativos que algunas constituciones conceden al Ejecutivo, el diseño de sistemas de partidos que configuran escenarios proclives a la intervención presidencial y la carencia de maestría suficiente de los legisladores para balancear al Ejecutivo (Alemán y Tsebelis, 2005; Jones et al., 2000; Mainwaring y Shugart, 1997). Otros autores, sin embargo, ofrecen una visión menos extremista de las relaciones entre el Ejecutivo y el Legislativo y sugieren que los parlamentos latinoamericanos participan en el proceso de toma de decisiones, aunque su influencia sea limitada porque no logran establecer un contrapunto al poder presidencial (Morgenstern y Nacif, 2001).

Los estudiosos de la región a menudo argumentan que los parlamentos son reactivos y que su actuación es importante en la medida en que su cooperación con el Ejecutivo facilita o no la gobernabilidad (Cox y Morgenstern, 2001). Al mismo tiempo, los trabajos empíricos sobre política legislativa han evidenciado una importante diversidad, por lo que resultaría un error considerar a todos los congresos latinoamericanos como actores sin iniciativa y a todos los presidentes como actores dominantes (Alcántara et al., 2005; Calvo, 2008; García Montero, 2009; Huneeus y Berríos, 2005; Saeigh, 2010).

Este artículo ofrece un aporte a esta línea de investigación, desarrollando un análisis comparado de la participación presidencial en la producción legislativa de cada país. El trabajo está centrado en los períodos gubernamentales de 30 presidentes latinoamericanos que ocuparon el cargo entre 1993 y 2010. Debido al limitado número de casos, la principal estrategia empírica es un análisis cualitativo comparativo (QCA). Los resultados del análisis destacan que la combinación de un diseño institucional favorable al Ejecutivo y una división del Congreso en bloques ideológicos bien diferenciados favorece el control presidencial de la agenda legislativa. Otras dos condiciones, la existencia de una mayoría parlamentaria afín y la ausencia de fragmentación partidaria, se combinan con estos elementos para garantizar el predominio del presidente.

\footnotetext{
Los argumentos para estas críticas son principalmente tres. El más importante de ellos es que en las democracias modernas el procedimiento legislativo es impulsado en primer lugar por el gobierno, la segunda crítica es que las leyes aprobadas de iniciativas presidenciales son las de mayor importancia y trascendencia (Molas y Pitarch, 1987: 143) y, por último, que la tasa de aprobación de los proyectos presentados por los congresistas es mucho menor que la del Poder Ejecutivo.
} 
El artículo está estructurado en cinco partes. En la primera sección se establece un criterio comparativo para evaluar el control presidencial de la agenda y se define la variable dependiente. La segunda sección identifica las posibles causas del predominio presidencial y operacionaliza los conceptos centrales. En la tercera sección se ofrece un análisis comparativo que identifica la existencia de tres contextos institucionales: uno en el que los presidentes tienen prácticamente garantizado el control de la agenda, otro en el que este control es en ocasiones viable y un tercero en el que resulta casi imposible para el presidente dominar la producción legislativa. El examen de la tabla de verdad permite identificar las condiciones iniciales que garantizan el control ejecutivo de la agenda. En la cuarta sección extendemos el procedimiento, identificando las condiciones que hacen el control de la agenda inviable, posible, y probable. Un análisis estadístico complementario, empleando un modelo saturado, permite verificar estos resultados cuando se trata la variable dependiente como una medida continua. Las conclusiones enfatizan el significado de estas configuraciones institucionales para la calidad de la democracia y la gobernabilidad, destacando que la eficiencia legislativa no entra en conflicto con el proceso democrático.

\section{EL CONTROL PRESIDENCIAL DE LA AGENDA LEGISLATIVA}

Por control de la agenda nos referimos a la capacidad del gobierno para monopolizar la iniciativa parlamentaria o limitar el rango de proyectos aprobados por el Poder Legislativo. La literatura sobre el control de la agenda en América Latina ha analizado áreas específicas de política pública, como la formulación del presupuesto o la adopción de legislación electoral (Baldez y Carey, 1999; Alemán y Tsebelis, 2005), pero sin ofrecer un indicador general para medir el peso del Ejecutivo en la producción parlamentaria.

Para medir el control del gobierno sobre la agenda este artículo emplea la participación legislativa del presidente, definida como la proporción de leyes iniciadas por el Ejecutivo sobre el total de legislación aprobada durante un período presidencial (García Montero, 2007: 77). Esta medida se diferencia de los porcentajes de éxito legislativo del presidente, el indicador de capacidad ejecutiva más comúnmente utilizado (García Montero, 2009; Saiegh, 2010; 2011; Steger, 1997). Una abundante producción académica, mayoritariamente estudios de caso, ${ }^{2}$ ha tomado como variable dependiente las tasas de aprobación logradas por los presidentes para evaluar su grado de control sobre la legislación y la cooperación legislativa. Aunque no tan numerosos, también existen trabajos de corte comparado centrados en el éxito legislativo de los presidentes de distintos países. ${ }^{3}$

2 Para el caso de México se puede consultar Casar (2002), Hurtado (2002), Nava y Yáñez (2003) o Weldon (2004); para el caso brasileño Figueiredo y Limongi (2000), Ricci (2002); Samuels (2002) o Amorim y Santos (2003); para Argentina Molinelli et al. (1999), Mustapic (2002), Calvo (2004) o Alemán y Calvo (2006), Alemán y Navia (2010); para el caso chileno, Siavelis (2002); Alemán y Navia (2010); para Colombia Cárdenas et al. (2008); para Honduras, Taylor y Díaz (1999); para Nicaragua, Navarro (2004); para los países centroamericanos, Ajenjo (2005); para Ecuador, Mejía (2000); para Uruguay Lanzaro et al. (2000) o Magar y Moraes (2007); para Paraguay se puede ver Molinas et al. (2004) y, para el caso venezolano, Amorim y Malgar (2000).

3 Veáse Saiegh (2011); Figueiredo et al. (2004), Alcántara et al. (2005); Alcántara y García (2008); García (2009) o Figueiredo et al. (2011) 
Mientras que el indicador empleado en este artículo (la tasa de participación legislativa) refleja la probabilidad de que una ley aprobada haya sido iniciada por el Ejecutivo, el indicador comúnmente empleado en la literatura (la tasa de éxito legislativo) refleja la probabilidad de que una ley iniciada por el presidente sea aprobada por el Parlamento. Estas dos variables capturan así aspectos diferentes de la influencia presidencial: la capacidad del Ejecutivo para controlar la agenda influye sobre la coherencia de las políticas públicas y la eficiencia del proceso legislativo (Carroll y Pachón, 2012), mientras que las tasas de éxito reflejan condiciones generales para la gobernabilidad (Saiegh, 2011).

Este trabajo argumenta que no es acertado considerar a los parlamentos latinoamericanos como instituciones mayoritariamente reactivas en el cumplimiento de la función legislativa. Si bien los presidentes presentan altas tasas de éxito legislativo en distintos países de la región, ${ }^{4}$ ello no prueba que no deban negociar la aprobación de sus propuestas con el órgano legislativo, ni que el Parlamento esté estrictamente supeditado a sus iniciativas. Menos de la mitad de los presidentes incluidos en nuestra muestra lograron una participación mayoritaria en la producción de leyes, mientras que más del 75\% de los presidentes analizados por Saiegh (2011) obtuvieron respaldo legislativo para la mayoría de sus proyectos de ley. Esto sugiere que pocos gobiernos consiguen dominar la agenda del Parlamento, a pesar de que las tasas de éxito de los presidentes son consistentemente mayores que las de los legisladores. ${ }^{5}$ En línea con nuestro argumento, García $(2007 ; 2009)$ ha mostrado empíricamente que bajo determinadas circunstancias los parlamentos son promotores de legislación, por lo que no es acertado mantener que estas instituciones tienen un papel irrelevante en la iniciativa legislativa. De ahí que resulte pertinente utilizar un indicador que capture el control de la agenda de forma más adecuada.

La Figura 1 muestra el porcentaje de proyectos iniciados por el presidente del total de leyes aprobadas durante 30 administraciones latinoamericanas en el período 1993-2010. Nuestra muestra incluye todos los gobiernos de la región, para ello tenemos información completa referente a la variable dependiente y a las variables independientes (presentadas en la sección siguiente), siendo representativa de la variabilidad regional así como de los distintos valores adoptados por las variables utilizadas ${ }^{6}$. El análisis abarca trece países de la región: Argentina (3 administraciones), Bolivia (2), Brasil (1), Chile (4),

\footnotetext{
Alcántara y García (2008).

Es habitual que los diputados presenten una gran cantidad de proyectos de ley, muchos de estos son para "agradar a votantes o grupos de interés" (Mollinelli et al., 1999: 97), teniendo una baja probabilidad de ser aprobados. A pesar de ello, los legisladores los presentan siendo instrumentalizados para hacerse visibles ante sus electores. El Ejecutivo, por el contrario, introduce una menor cantidad de proyectos, analizando previamente la posible reacción del Legislativo para conseguir su apoyo, por lo que su tasa de éxito es superior (García, 2007: 7; Steger, 1997).

6 Los datos de participación presidencial no pudieron reconstruirse de manera sistemática para Guatemala, Nicaragua, y República Dominicana, por lo que estos países no fueron incluidos en el análisis. Adicionalmente, el uso de la Encuesta de Elites Parlamentarias (PELA) de la Universidad de Salamanca para medir la polarización ideológica restringe nuestro estudio al período que comienza en 1993. Desafortunadamente, la información de PELA es incluso posterior para los casos de Brasil (disponible desde 2003), Colombia (1998) y Panamá (1999). Estas limitaciones restringen el número de administraciones que han podido ser consideradas en el estudio.
} 
Figura 1. Participación de 30 presidentes en la producción legislativa

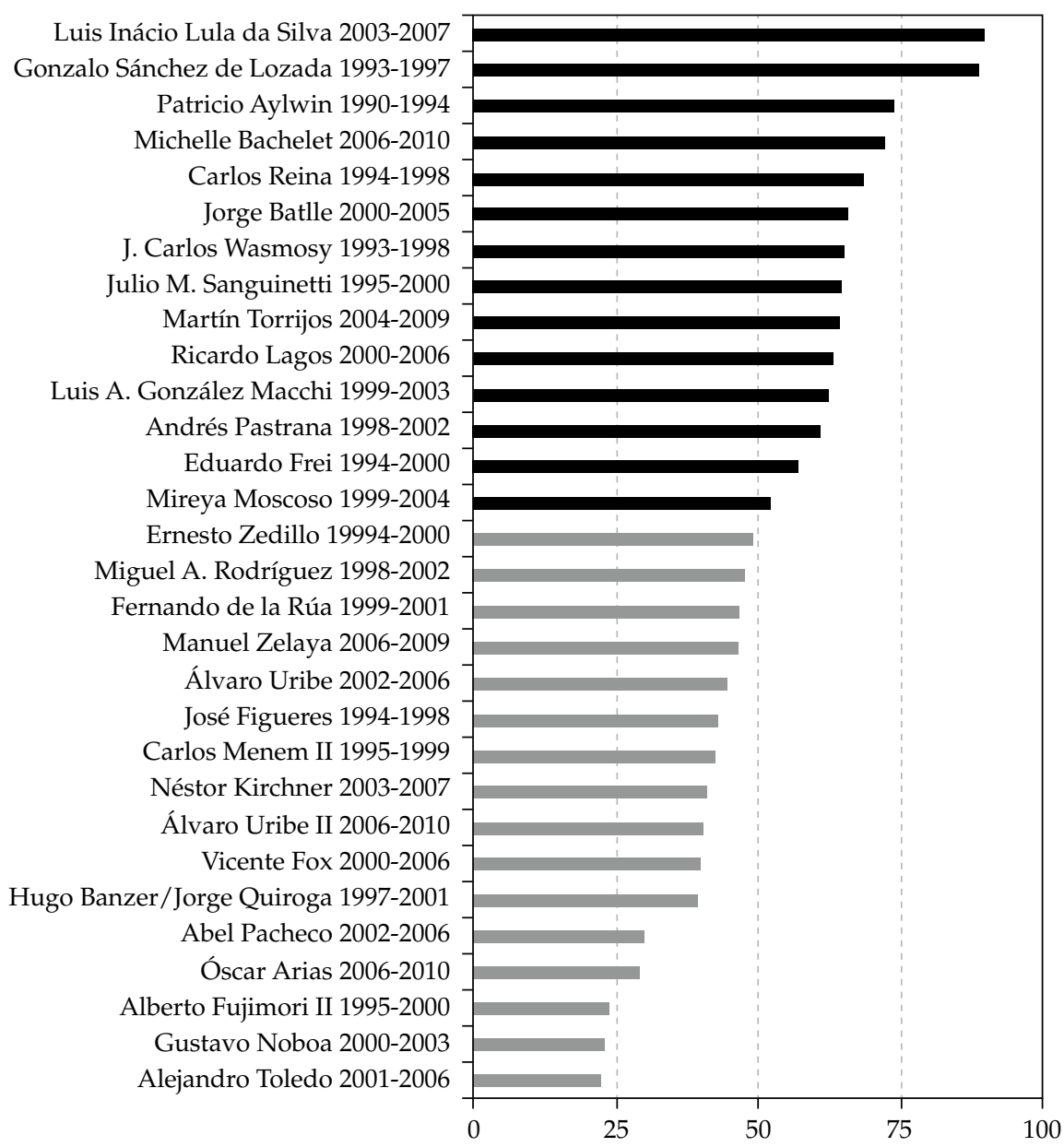

Fuente: Elaboración propia.

Colombia (3), Costa Rica (4), Ecuador (1), Honduras (2), México (2), Panamá (2), Perú (2), Paraguay (2) y Uruguay (2). ${ }^{7}$

La decisión de tomar como unidad de análisis los periodos presidenciales refleja el supuesto de que la actuación legislativa del presidente está condicionada por el contexto institucional y legislativo en que su actividad se desenvuelve. Si se tomara como unidad de análisis el país, sería difícil evaluar el papel que las características cambiantes de la legislatura tienen sobre el control presidencial de la agenda. Por otra parte, si se tomara

7 De la muestra excluimos los gobiernos de Valentín Paniagua en Perú (2000-01) y de Raúl Cubas Grau en Paraguay (1998-99), por su corta duración y por su carácter excepcional. La inclusión de estos casos no altera las conclusiones generales del estudio. 
como unidad de análisis el año legislativo, las observaciones no serían independientes entre sí, debido a que las condiciones causales se mantienen constantes durante los años en que la composición partidaria y las características institucionales de la legislatura permanecen inalteradas. ${ }^{8}$

El gráfico indica claramente que la variación entre diferentes administraciones no es despreciable. Incluso presidentes de un mismo país, como Gonzalo Sánchez de Lozada y Hugo Banzer, muestran a veces tasas de participación muy distintas, lo que destaca la importancia de tomar como unidad de análisis el período presidencial y no el país. La participación del presidente en la producción legislativa ha fluctuado entre $22 \%$ (en el caso de Alejandro Toledo en Perú) y $90 \%$ (en el caso del primer gobierno de Lula en Brasil). Nuestra pregunta de investigación se centra en explicar la capacidad de ciertos presidentes para dominar la iniciativa parlamentaria, por lo que a los efectos del análisis clasificamos las administraciones en dos grupos: aquellas que lograron un control mayoritario sobre la agenda legislativa (las 14 identificadas por barras oscuras en la Figura 1) y aquellas que no lo hicieron (las 16 restantes). Si bien la distribución de la variable es relativamente bimodal (las principales concentraciones de casos se producen entre $40 \%$ y $50 \%$, y entre 60 y $70 \%$ ), lo que justifica el uso de una clasificación dicotómica, la sección 4.3 de este artículo muestra que los resultados del análisis no cambian si analizamos la participación legislativa de los presidentes como una variable continua.

\section{CONDICIONES CAUSALES QUE AFECTAN EL CONTROL DE LA AGENDA}

¿Qué factores explican esta variación? Los pocos estudios que abordan este tema (Carroll y Pachón, 2012; García Montero, 2009) destacan algunos factores que pueden afectar la influencia del Ejecutivo sobre la actividad parlamentaria: el diseño institucional, la composición política del Parlamento y la naturaleza de los partidos o coaliciones. Los ejecutivos mantienen mayor influencia sobre la agenda cuando cuentan con ventajas institucionales, del mismo modo que los congresos muestran mayor capacidad proactiva cuando actúan en diseños institucionales que les favorecen. Sin embargo, la evidencia sugiere que es preciso tener en cuenta el efecto combinado de los factores institucionales y la conformación de mayorías partidarias.

Sobre la base de estos trabajos previos, el análisis se concentra en cuatro posibles explicaciones: las reglas institucionales del proceso legislativo, los poderes partidarios del presidente, la fragmentación del Parlamento y la distancia ideológica entre los bloques políticos. Un análisis formal sugiere, sin embargo, que estas variables interactúan para delimitar la influencia del Ejecutivo (Pérez-Liñán y Rodríguez Raga, 2009). El diseño institucional favorable al Ejecutivo puede tener poca relevancia cuando el partido de

8 Debido a que nuestras variables independientes cambian apenas con cada período legislativo o cada reforma constitucional, el uso de países-año como unidades de análisis en un modelo de regresión (ver sección 4.3) aumentaría artificialmente el número de observaciones e induciría a autocorrelación en los residuos. 
gobierno cuenta con sólidas mayorías parlamentarias o cuando todos los partidos tienen preferencias ideológicas similares. Por el contrario, los poderes constitucionales del Ejecutivo pueden tener un impacto mayor si el Congreso se encuentra altamente fragmentado. Para entender las consecuencias de la interacción de estas variables es preciso analizar sus posibles configuraciones causales, más allá de sus efectos "netos"sobre la influencia del Ejecutivo (Ragin, 2008; Schneider y Wagemann, 2012).

\section{Las reglas institucionales (IPIL)}

Esta investigación sostiene que cuando las reglas institucionales son más beneficiosas para el Ejecutivo, aumenta la probabilidad de que el presidente tenga una participación dominante en la actividad legislativa. El proceso legislativo está marcado por las reglas institucionales establecidas en la Constitución y en los reglamentos internos del Parlamento. Los pasos establecidos por este diseño configuran escenarios que pueden ser más beneficiosos para uno u otro poder.

Diversos estudios han evaluado los poderes constitucionales del presidente, destacando la distinción entre el poder de agenda positivo (proactivo) y negativo (reactivo) (Cox, 2006; García Montero, 2009; Mainwaring y Shugart, 1997; Metcalf, 2000; Negretto, 2013; Payne et al., 2003; PNUD, 2005; Samuels y Shugart, 2003; Shugart y Carey, 1992; Shugart y Haggard, 2001). El análisis de casos históricos sugiere que el control presidencial de la agenda se vea influenciado no solamente por los poderes reactivos del Ejecutivo (Baldez y Carey, 1999), sino también por sus poderes proactivos (Alemán y Tsebelis, 2005) y por la naturaleza del proceso parlamentario (Steger, 1997). Por esta razón, tomamos como indicador de capacidad institucional ejecutiva el Índice de Potencia Institucional Legislativa (IPIL) elaborado por García Montero (2009, capítulo 4).

Este índice IPIL refleja no solamente las normas constitucionales sino también el procedimiento parlamentario, integrando 14 criterios institucionales que permiten evaluar en qué medida un Ejecutivo tiene mayor poder de agenda. La información para los 14 ítems es agregada en cinco dimensiones, con el fin de capturar el rol del presidente en las tres etapas del proceso legislativo (de iniciativa, constitutiva y de eficacia), así como el efecto del bicameralismo y los procedimientos legislativos extraordinarios. Estos cinco componentes son luego promediados para identificar un valor integrado. La Figura 2 resume gráficamente la estructura de agregación de los componentes del índice.

El rango posible para este índice varía entre 0 y 1 ; los valores cercanos a 1 indican que el presidente cuenta con mecanismos institucionales que favorecen su participación en la actividad legislativa, mientras que los valores próximos a 0 reflejan una mayor influencia institucional del Parlamento (García Montero, 2009: 183). En nuestra muestra de administraciones el valor mínimo observado es de 0,32 (en Costa Rica y México) y el máximo de 0,71 (en Chile), con una media de 0,46 . A los efectos del análisis comparativo consideramos a los presidentes con valores mayores a 0,5 como ejecutivos con una alta potencia institucional. 
Figura 2. Índice de Potencia Institucional Legislativa (IPIL)

Iniciativa exclusiva; capacidad de influencia en la elaboración del orden del día o la agenda discusión; tipo de mayoría necesaria para modificar el orden del día

Capacidad del presidentes para forzar el tratamiento de urgencia; forma de integración de las comisiones; prerrogativas para evitar el tratamiento de la ley en comisión; poder de las comisiones para el tratamiento de los proyectos de ley

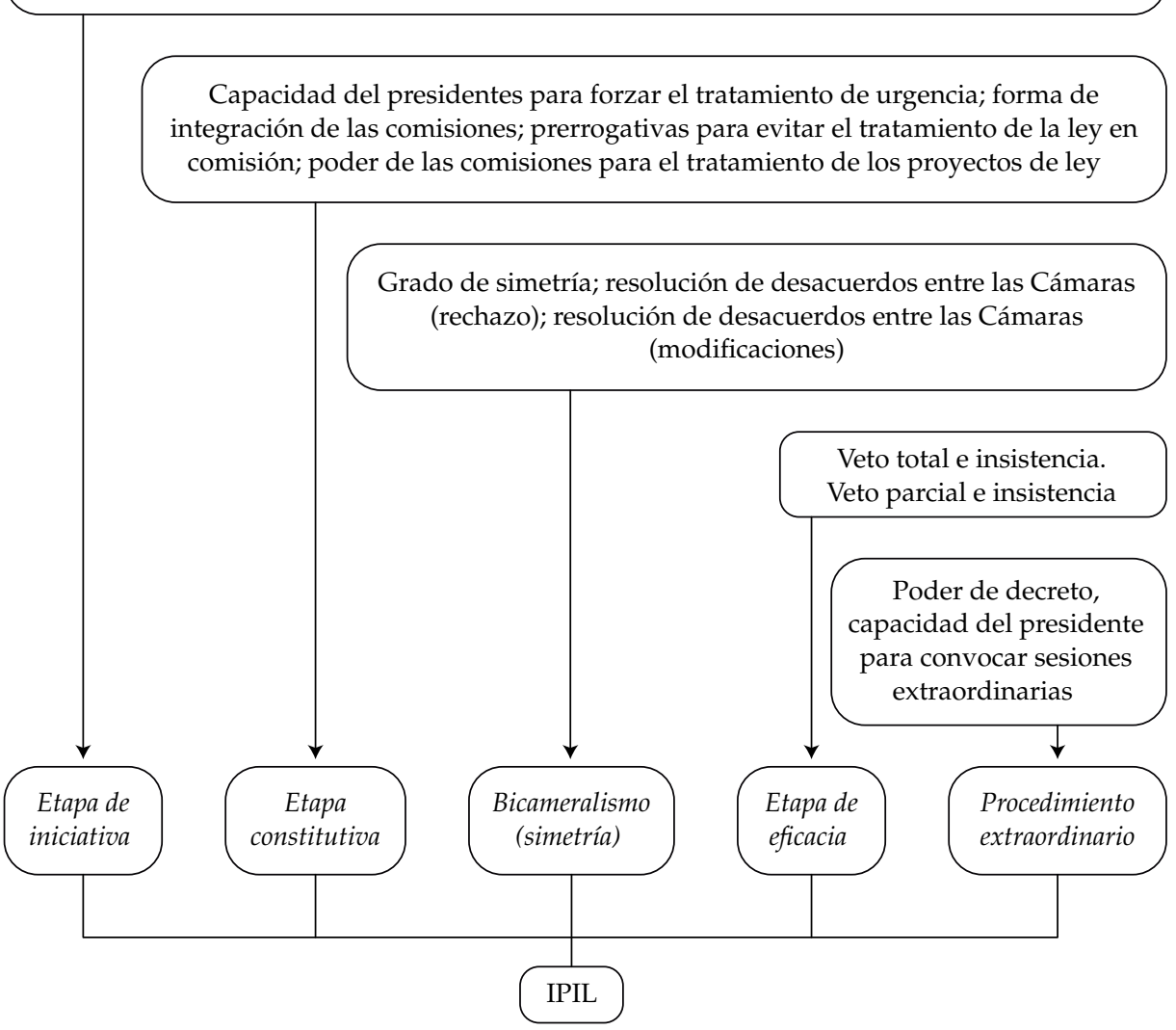

Fuente: elaboración propia a partir de García Montero (2009, capítulo 4).

\section{Mayorías parlamentarias}

La literatura ha enfatizado que los presidentes que no poseen una mayoría en el Parlamento cuentan con mayores dificultades para controlar la agenda legislativa (Linz, 1990; Mainwaring, 1993; Mainwaring y Shugart, 1997; Steger, 1997). Esta idea ha inspirado numerosas hipótesis en el contexto latinoamericano. Así, se ha afirmado que los gobiernos de minoría incentivan el uso de los poderes constitucionales unilaterales por parte del Ejecutivo (Ferreira y Goretti, 1996; Cox y Morgenstern, 2001); ${ }^{9}$ potencian el poder del "partido mediano" para definir la modificación del status quo (Nacif, 2005;

9 Cuando un presidente cuenta con una mayoría legislativa amplia el uso del poder unilateral de decreto es poco relevante para alcanzar sus metas políticas (Cox y Morgenstern, 2001) e, inversamente, cuando la mayoría del Congreso confía en el presidente como un agente, puede delegar poder de decreto en el Ejecutivo para apresurar la agenda legislativa (Carey y Shugart, 1998; Pérez-Liñán y Rodríguez Raga, 2009). 
Negretto, 2002) ${ }^{10}$ y agudizan la necesidad de formar coaliciones para gobernar (Altman, 2001; Chasquetti, 2001; 2008; Amorim, 1998; Pérez Liñán, 2000). Todas estas condiciones afectan la influencia del presidente en la agenda, y le imponen diversas estrategias para superar su situación minoritaria. ${ }^{11}$

Para construir un indicador sencillo que refleje la posición del presidente en el Congreso se ha tomado el porcentaje de escaños que obtuvo el partido del presidente en la Asamblea (o su coalición, si varios partidos integraron el gobierno). ${ }^{12}$ En sistemas bicamerales se han promediado los porcentajes para la Cámara Baja y la Cámara Alta. El rango observado para esta variable fluctúa entre el 12,8\% (Uribe I) y el 79,5\% (Banzer). Con fines comparativos nos referimos a gobiernos de mayoría cuando su bloque parlamentario controla en promedio más del $50 \%$ de las curules.

\section{Fragmentación (número efectivo de partidos)}

Para fines analíticos es preciso distinguir el efecto de las mayorías parlamentarias de una variable correlacionada pero conceptualmente distinta, la fragmentación del sistema de partidos. Mainwaring (1993) fue uno de los primeros autores en señalar que el multipartidismo en contextos presidencialistas puede ser considerado como un factor potencialmente peligroso para la gobernabilidad y la estabilidad de las democracias. En este mismo sentido, Sartori (1999: 151) ha afirmado que "cuanto mayor sea el número de partidos (que tienen voz) mayor será la complejidad y probablemente complicación del sistema". En opinión de Stein et al. (2006: 53) es probable que un sistema muy fragmentado produzca una legislatura más activa, pero también puede convertirla en un foro en el que apenas se negocian beneficios particulares en lugar de políticas proactivas. A mayor número de partidos, mayores son los costos de transacción para el Ejecutivo y las posibles dificultades para controlar la agenda (Cox y McCubbins, 1999). García Montero (2009), sin embargo, sostiene que la fragmentación partidista es relevante para explicar el éxito legislativo del presidente, pero no su participación en la producción legislativa.

El indicador convencionalmente utilizado para medir la dispersión del poder político en el Legislativo es el número efectivo de partidos, definido como $N E P=\frac{1}{\sum p_{i}^{2}}$, en donde p representa la proporción de curules de cada partido i (Laakso y Taagepera, 1979). Un valor de 2,0 refleja la presencia de dos partidos de igual tamaño, mientras que

10 El teorema del votante medio predice que el resultado del procedimiento de toma de decisión en el Congreso convergerá alrededor del punto ideal del partido mediano. Negretto (2003) considera que la capacidad presidencial para sacar adelante su agenda legislativa depende de tres factores: la posición que ocupa el partido mediano, la posición del statu quo y los poderes presidenciales otorgados por la Constitución. Esta perspectiva se basa en Krehbiel (1998) y en Colomer y Negretto (2005).

11 Magar y Moraes (2007: 42) sostienen que en gobiernos divididos el apoyo de las cámaras a la agenda del Ejecutivo puede lograrse en tres formas: partidista (las coaliciones acuerdan sobre programas de gobierno), por soborno (de los partidos de la oposición o de legisladores individuales según cada cuestión), y por vía pública (cuando los gobiernos apelan a la opinión pública para que sea esta quien presione a los parlamentarios).

12 Los datos sobre coaliciones fueron extraídos de Chasquetti (2008) y actualizados a partir de fuentes periodísticas. 
un valor de 3,0 indica que la asamblea está dividida en tres bloques equivalentes. En nuestra muestra, los valores de NEP fluctúan entre 1,8 (México bajo Zedillo) y 7,2 (Brasil bajo Lula). ${ }^{13}$ Los sistemas de partidos son clasificados como fragmentados cuando el valor del NEP supera el umbral de 3,5.

\section{La distancia ideológica (polarización)}

Una última condición que puede afectar el resultado de interés es la distancia ideológica entre los principales partidos políticos. Una distancia ideológica significativa entre los partidos en el Congreso ocasiona mayor conflicto entre el Ejecutivo y el Legislativo y genera mayores incentivos para que los presidentes busquen controlar la agenda. Como afirman Mainwaring y Scully (1995: 33), existe una relación inversamente proporcional entre la polarización de un sistema de partidos y el entendimiento entre los poderes de gobierno.

Para medir esta condición causal utilizamos el índice de polarización ponderada, calculado a partir de la posición que cada partido ocupa en el espectro izquierda-derecha, y de su peso legislativo medido en escaños (Oñate y Ocaña, 1999: 42). La fórmula para calcular el índice es $I P P=\Sigma p_{i}\left(x_{i}-\Sigma p_{i} x_{i}\right)^{2}$, en donde $p_{\mathrm{i}}$ es la proporción de escaños de cada partido $i$ con representación parlamentaria y $x_{\mathrm{i}}$ representa la posición del partido en una escala ideológica que varía entre 1 (izquierda) y 10 (derecha). Para la elaboración del índice hemos extraído los datos de ubicación ideológica del Proyecto Elites Parlamentarias Latinoamericanas (PELA). ${ }^{14}$

El IPP refleja la media de las distancias entre el legislador promedio en el Congreso y la ubicación de cada partido (estas distancias son elevadas al cuadrado para ponderar las brechas mayores). Por ejemplo, en una asamblea hipotética conformada por dos partidos de igual tamaño, dos partidos centristas ubicados en las posiciones 5 y 6 de la escala ideológica generan un IPP $=0,25$. Si el partido de izquierda se mueve hacia la posición 4, aumentando la distancia ideológica, IPP $=1,00$. Y si el partido de derecha aumenta esta brecha desplazándose hacia la posición $7, \operatorname{IPP}=2,25$. En nuestra muestra de administraciones, el valor medio para el índice de polarización es de 1,16, con un mínimo de 0,11 y un máximo de 2,90. Consideramos como sistemas partidarios con una clara distancia ideológica aquellos en los que el índice de polarización adquiere valores superiores a 1,00 (lo que ocurre en 13 de los 30 casos).

La Tabla 1 recoge los valores de las variables independientes para los períodos de gobierno analizados. Las columnas correspondientes a cada variable explicativa reportan el valor del indicador cuantitativo empleado para capturar la información y su interpretación cualitativa de acuerdo con categorías discretas. Las cuatro condiciones analíticas invocadas para entender el control de la agenda parlamentaria son la presencia de un Ejecutivo

13 Para sistemas bicamerales empleamos la media de ambas cámaras.

14 Los valores de $x_{\mathrm{i}}$ fueron estimados como la media de la autoubicación ideológica de los legisladores de cada partido en la Cámara Baja. El proyecto PELA (Ref. SEC94/0284; SEC95/0845 y SEC02/3484), dirigido por Manuel Alcántara, lleva realizándose en las Cámaras Bajas de los países latinoamericanos desde 1994. Más información sobre la citada investigación puede verse en García y Mateos (2006). 
Tabla 1. Indicadores para variables independientes y condiciones causales

Administración IPIL Potencia $\begin{gathered}\text { Curules } \\ (\%)\end{gathered}$ Mayoría NEP Fragmentación Polarización $\begin{gathered}\text { Distancia } \\ \text { ideológica }\end{gathered}$

Presidentes sin control de la agenda legislativa

\begin{tabular}{lcccccccc}
\hline Toledo & 0,48 & $\mathrm{~N}$ & 46,7 & $\mathrm{~N}$ & 4,4 & $\mathrm{~S}$ & 0,58 & $\mathrm{~N}$ \\
Noboa & 0,59 & $\mathrm{~S}$ & 28,9 & $\mathrm{~N}$ & 5,7 & $\mathrm{~S}$ & 1,40 & $\mathrm{~S}$ \\
Fujimori II & 0,48 & $\mathrm{~N}$ & 45,8 & $\mathrm{~N}$ & 2,9 & $\mathrm{~N}$ & 1,56 & $\mathrm{~S}$ \\
Arias II & 0,32 & $\mathrm{~N}$ & 44,0 & $\mathrm{~N}$ & 3,3 & $\mathrm{~N}$ & 0,25 & $\mathrm{~N}$ \\
Pacheco & 0,32 & $\mathrm{~N}$ & 33,3 & $\mathrm{~N}$ & 4,7 & $\mathrm{~S}$ & 0,60 & $\mathrm{~N}$ \\
Banzer & 0,41 & $\mathrm{~N}$ & 79,5 & $\mathrm{~S}$ & 4,6 & $\mathrm{~S}$ & 1,16 & $\mathrm{~S}$ \\
Fox & 0,32 & $\mathrm{~N}$ & 36,4 & $\mathrm{~N}$ & 2,0 & $\mathrm{~N}$ & 2,18 & $\mathrm{~S}$ \\
Uribe II & 0,52 & $\mathrm{~S}$ & 63,0 & $\mathrm{~S}$ & 7,0 & $\mathrm{~S}$ & 0,80 & $\mathrm{~N}$ \\
Kirchner & 0,33 & $\mathrm{~N}$ & 53,6 & $\mathrm{~S}$ & 3,1 & $\mathrm{~N}$ & 0,72 & $\mathrm{~N}$ \\
Menem II & 0,33 & $\mathrm{~N}$ & 51,5 & $\mathrm{~S}$ & 2,7 & $\mathrm{~N}$ & 0,39 & $\mathrm{~N}$ \\
Figueres & 0,32 & $\mathrm{~N}$ & 49,1 & $\mathrm{~N}$ & 3,3 & $\mathrm{~N}$ & 0,85 & $\mathrm{~N}$ \\
Uribe I & 0,52 & $\mathrm{~S}$ & 12,8 & $\mathrm{~N}$ & 3,2 & $\mathrm{~N}$ & 1,00 & $\mathrm{~N}$ \\
Zelaya & 0,39 & $\mathrm{~N}$ & 48,0 & $\mathrm{~N}$ & 2,4 & $\mathrm{~N}$ & 0,76 & $\mathrm{~N}$ \\
De la Rúa & 0,33 & $\mathrm{~N}$ & 39,4 & $\mathrm{~N}$ & 2,9 & $\mathrm{~N}$ & 0,77 & $\mathrm{~N}$ \\
Rodríguez & 0,32 & $\mathrm{~N}$ & 47,4 & $\mathrm{~N}$ & 2,6 & $\mathrm{~N}$ & 0,80 & $\mathrm{~N}$ \\
Zedillo & 0,32 & $\mathrm{~N}$ & 60,5 & $\mathrm{~S}$ & 1,8 & $\mathrm{~N}$ & 0,65 & $\mathrm{~N}$ \\
\hline
\end{tabular}

Presidentes que dominaron la agenda legislativa

\begin{tabular}{lcccccccc}
\hline Moscoso & 0,62 & $\mathrm{~S}$ & 39,4 & $\mathrm{~N}$ & 3,3 & $\mathrm{~N}$ & 2,03 & $\mathrm{~S}$ \\
Frei & 0,71 & $\mathrm{~S}$ & 50,0 & $\mathrm{~N}$ & 5,1 & $\mathrm{~S}$ & 2,28 & $\mathrm{~S}$ \\
Pastrana & 0,52 & $\mathrm{~S}$ & 23,5 & $\mathrm{~N}$ & 3,3 & $\mathrm{~N}$ & 1,44 & $\mathrm{~S}$ \\
González Macchi & 0,33 & $\mathrm{~N}$ & 54,8 & $\mathrm{~S}$ & 2,3 & $\mathrm{~N}$ & 0,59 & $\mathrm{~N}$ \\
Lagos & 0,71 & $\mathrm{~S}$ & 47,5 & $\mathrm{~N}$ & 5,7 & $\mathrm{~S}$ & 2,61 & $\mathrm{~S}$ \\
Torrijos & 0,62 & $\mathrm{~S}$ & 53,9 & $\mathrm{~S}$ & 2,9 & $\mathrm{~N}$ & 1,55 & $\mathrm{~S}$ \\
Sanguinetti & 0,48 & $\mathrm{~N}$ & 61,6 & $\mathrm{~S}$ & 3,3 & $\mathrm{~N}$ & 0,75 & $\mathrm{~N}$ \\
Wasmosy & 0,33 & $\mathrm{~N}$ & 46,0 & $\mathrm{~N}$ & 1,9 & $\mathrm{~N}$ & 0,11 & $\mathrm{~N}$ \\
Batlle & 0,48 & $\mathrm{~N}$ & 55,2 & $\mathrm{~S}$ & 3,2 & $\mathrm{~N}$ & 1,67 & $\mathrm{~S}$ \\
Reina & 0,39 & $\mathrm{~N}$ & 55,5 & $\mathrm{~S}$ & 2,0 & $\mathrm{~N}$ & 0,35 & $\mathrm{~N}$ \\
Bachelet & 0,71 & $\mathrm{~S}$ & 53,0 & $\mathrm{~S}$ & 5,8 & $\mathrm{~S}$ & 2,90 & $\mathrm{~S}$ \\
Aylwin & 0,71 & $\mathrm{~S}$ & 49,2 & $\mathrm{~N}$ & 5,0 & $\mathrm{~S}$ & 1,75 & $\mathrm{~S}$ \\
Sánchez de Lozada I & 0,41 & $\mathrm{~N}$ & 61,9 & $\mathrm{~S}$ & 2,9 & $\mathrm{~N}$ & 0,88 & $\mathrm{~N}$ \\
Lula I & 0,52 & $\mathrm{~S}$ & 61,3 & $\mathrm{~S}$ & 7,2 & $\mathrm{~S}$ & 1,35 & $\mathrm{~S}$ \\
\hline
\end{tabular}

Fuente: Elaboración propia a partir de García Montero (2009), composición de las Cámaras y PELA.

Notas: $\mathrm{N}$ indica la ausencia de un atributo discreto y $\mathrm{S}$ su presencia. Los umbrales para clasificar los casos son 0,5 (IPIL), 50\% (Curules), 3,5 (Número Efectivo de Partidos), y 1,0 (Polarización). Las presidencias están listadas en función de su participación en la producción legislativa, en orden ascendiente. Los casos en el panel inferior tienen tasas de participación superiores al 50\% (controlaron la agenda). 
con potencia institucional, de una mayoría parlamentaria en favor del presidente, de un sistema de partidos fragmentado y de una clara distancia ideológica entre los bloques políticos. El uso de estas categorías discretas en lugar de las variables continuas permite evaluar el efecto de cuatro condiciones causales conceptualmente bien definidas, en lugar de confiar la explicación a los momentos estadísticos de los indicadores continuos, cuya representatividad está restringida por el limitado número de casos. ${ }^{15}$

\section{ANÁLISIS CUALITATIVO COMPARATIVO}

La Tabla 1 sugiere que ninguna de estas cuatro condiciones es estrictamente necesaria para que el presidente domine la agenda parlamentaria. Los 14 casos de control de agenda identificados en la Figura 1 no comparten ninguna característica común, lo que indica que los presidentes pueden alcanzar una posición dominante en ausencia de cualquiera de estas condiciones (Schneider y Wagemann, 2012: 69-75).

¿Existe, no obstante, alguna combinación causal que asegure a los presidentes el control del proceso legislativo? Para responder a esta pregunta desarrollamos en esta sección un análisis cualitativo comparativo (QCA, por su sigla en inglés). Cinco razones justifican la elección de QCA como principal método en este artículo. En primer lugar, QCA permite analizar sistemáticamente todas las combinaciones posibles de condiciones causales con el fin de identificar mecanismos de causalidad coyuntural, es decir, configuraciones de factores que resultan suficientes para producir el resultado de interés. Por contraste, el análisis estadístico convencional busca estimar del efecto "neto" de cada variable independiente, controlando por las demás. Sabemos sin embargo que los factores institucionales operan de manera interactiva. Por ejemplo, el poder constitucional del presidente resulta poco relevante cuando no existe distancia ideológica entre el Ejecutivo y el Legislativo. En segundo lugar, si bien la tasa de participación presidencial en la legislación es una variable continua, nuestra pregunta de investigación refiere conceptualmente a una distinción categórica. Se trata de explicar por qué algunos presidentes dominan la mayoría de la legislación aprobada en sus países y otros no. Esto sugiere la conveniencia de emplear una variable dependiente dicotómica, calibrada en el umbral de 50\%. En tercer lugar, el uso de un número intermedio de casos $(\mathrm{n}=30)$ limita el uso eficiente de estimadores estadísticos, en especial los estimadores de máxima verosimilitud que corrigen por la naturaleza limitada de nuestra variable dependiente (ya sea que esta se defina como una variable dicotómica o como un porcentaje). En cuarto lugar, el análisis de QCA permite realizar un tratamiento sistemático de los contrafácticos -es decir, las configuraciones causales para las cuales no existen observaciones- que no resulta posible en el análisis cuantitativo. Finalmente, algunos estudios anteriores (v.g., García Montero, 2009) han empleado técnicas estadísticas para explorar este tema, por lo que QCA ofrece una

15 La calibración de las variables a partir de criterios teóricos permite también emplear un análisis de conjuntos difusos (Ragin, 2008). El artículo se concentra en el análisis de conjuntos discretos para facilitar la interpretación sustantiva de los resultados, incluyendo la representación gráfica de la tabla de verdad en la Figura 3. 
oportunidad para abordar el problema desde otra perspectiva, permitiendo identificar nuevos resultados. ${ }^{16}$

Con el fin de presentar el análisis de QCA de manera intuitiva, la Figura 3 identifica todas las combinaciones posibles de condiciones causales y compara las administraciones observadas de acuerdo con este esquema. Las cuatro variables dicotómicas generan 16 configuraciones causales, representadas por medio de un plano de Cronqvist (una forma estilizada de diagrama de Venn). El rectángulo más amplio simboliza el conjunto de los 30 presidentes estudiados. En la mitad derecha se encuentran todas las administraciones con potencia institucional, y en la mitad izquierda aquellas sin potencia. En la mitad inferior se encuentran las administraciones respaldadas por una mayoría legislativa, y en la mitad superior aquellas en minoría. Las dos condiciones restantes se representan como rectángulos internos en el plano. Todos los casos de fragmentación partidaria se

Figura 3. Representación gráfica de la tabla de verdad

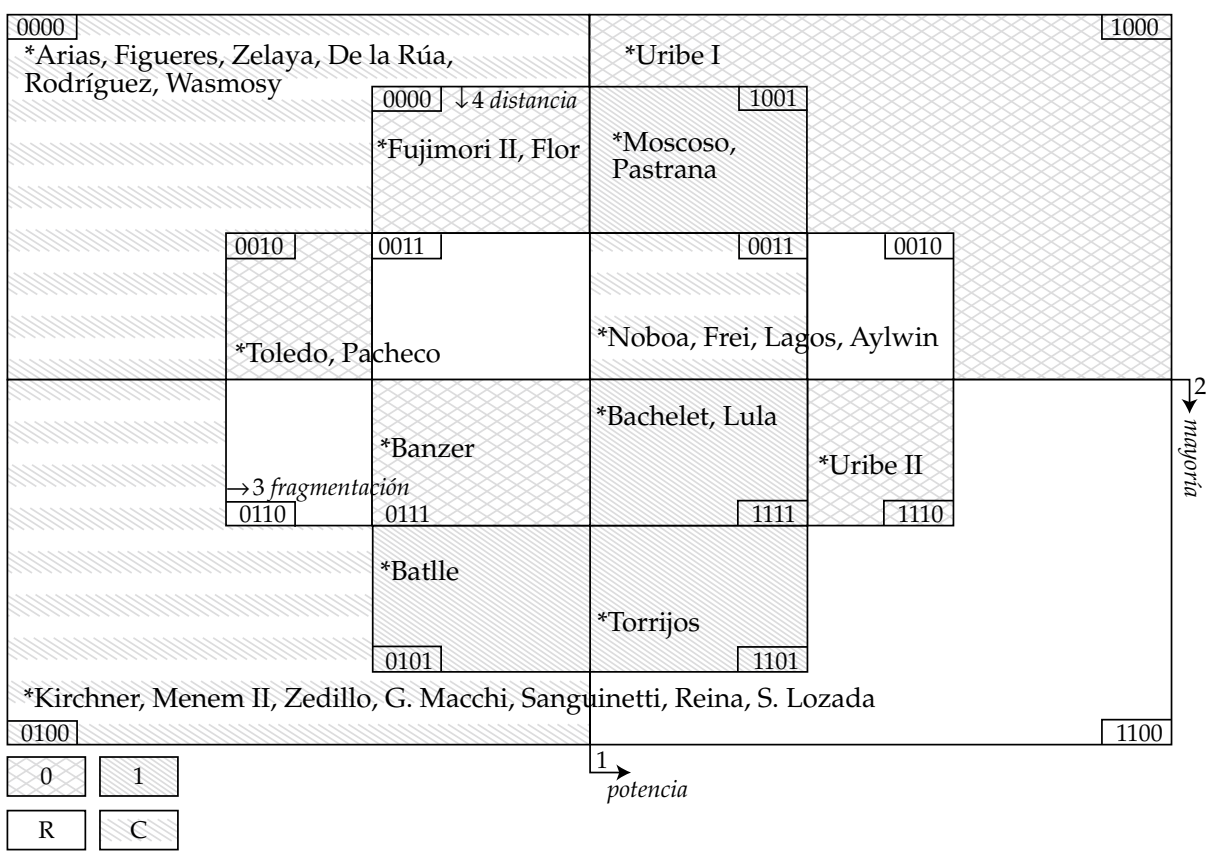

Nota: Las áreas marcadas con el rayado 0 corresponden a configuraciones sin control de la agenda (falsas), y las marcadas con 1 a configuraciones en las que todos los presidentes controlan la agenda (verdaderas); $\mathrm{C}$ corresponde a configuraciones contradictorias (con resultados mixtos) y $\mathrm{R}$ a residuos. La referencia en la esquina de cada tipo indica el código de identificación en la Tabla 2.

16 Para verificar los resultados desde una perspectiva cuantitativa, en la sección 4.3 empleamos un diseño estadístico que busca replicar, mediante el uso de un modelo saturado, la especificación de QCA. El modelo saturado se basa en una ecuación de mínimos cuadrados que incluye todas las interacciones posibles entre las variables independientes y por lo tanto asegura una función lineal, para ello los valores esperados nunca se encuentran por debajo de 0 o por encima de 100. 
encuentran dentro del rectángulo horizontal, y los casos de distancia ideológica dentro del rectángulo vertical. La superposición de estos recuadros divide al plano en tantos polígonos como tipos posibles. Cada uno de ellos se halla identificado por un código binario que refleja la presencia o ausencia de las condiciones explicativas. En el ángulo superior izquierdo, por ejemplo, la celda 0000 corresponde a situaciones caracterizadas por la ausencia de potencia legislativa, de mayorías parlamentarias, de fragmentación partidaria y de distancia ideológica; mientras que la celda contigua 0001 representa situaciones similares, pero en las que existe distancia ideológica en el Parlamento.

La variable dependiente está representada en la Figura 3 por medio del sombreado de cada celda. Las áreas marcadas con el rayado oscuro [0] corresponden a situaciones en las que ningún presidente logró el control de la agenda, mientras que las áreas marcadas con el rayado claro [1] representan configuraciones suficientes en donde todos los presidentes lograron controlar la agenda. Adicionalmente, los campos marcados con cuadriculado gris [C] corresponden a contextos con resultados contradictorios y los campos en blanco $[R]$, a situaciones residuales para las que no hay ejemplos históricos.

Cuatro situaciones identificadas en la Figura 3 parecen asegurar el control de la agenda parlamentaria: 1111 (Bachelet y Lula), 1101 (Torrijos), 1001 (Moscoso y Pastrana) y 0101 (Batlle). Pero esta lista de configuraciones suficientes puede ser simplificada. Consideremos, por ejemplo, las dos primeras configuraciones (1111 y 1101): en contextos en los que el presidente ha contado con potencia institucional, con una mayoría parlamentaria, y con una distancia ideológica suficientemente amplia frente a los partidos de oposición, el Ejecutivo ha dominado la agenda parlamentaria sin importar si el sistema de partidos se encuentra fragmentando o no.

A partir de este principio, el proceso de minimización lógica permite la reducción de estos cuatro tipos a tres situaciones: (1) el presidente tiene potencia institucional, una mayoría parlamentaria, y existe distancia ideológica en el Parlamento; (2) el presidente tiene potencia institucional y existe distancia ideológica en el Parlamento, pero el sistema de partidos no está fragmentado (lo que asegura el éxito aunque el Ejecutivo no cuente con una mayoría); o (3) el presidente cuenta con una mayoría parlamentaria y existe distancia ideológica en el Parlamento, pero el sistema no está fragmentado (lo que compensa que el Ejecutivo no tenga potencia ideológica). Estos implicantes primarios pueden representarse formalmente como:

distancia*potencia*mayoría

distancia* potencia* $\sim$ fragmentación

distancia*mayoría* fragmentación

en donde * representa la operación de conjunción $(y)$, en tanto $\sim$ representa la operación de negación (ausencia de). La distancia ideológica aparece como un elemento común a todas las situaciones que justifican el predominio del Ejecutivo. Pero para lograr este predominio el presidente debe contar además con potencia institucional o con una mayoría parlamentaria (en contextos favorables en los que la política legislativa no está 
fragmentada), o movilizar ambas capacidades en situaciones en que debe superar la fragmentación del sistema de partidos.

La Figura 3 muestra que el primer implicante primario (distancia*potencia*mayoría) cubre tres de los 14 casos de dominio presidencial (Bachelet, Lula y Torrijos); el segundo implicante (distancia*potencia* fragmentación), cubre otros tres (Moscoso, Pastrana y Torrijos) y el tercero (distancia*mayoría* fragmentación) cubre solo dos (Batlle y Torrijos). Debido a que la administración Torrijos se ve incluida en todas las soluciones, los tres implicantes en conjunto explican el 43\% de los casos de interés.

Para extender este análisis, la Tabla 2 representa los detalles de la Figura anterior bajo el formato de una tabla de verdad. Cada fila representa una de las 16 configuraciones causales posibles, identificadas por su código en la primera columna de la izquierda, y por sus atributos en las cuatro columnas siguientes.

En el panel derecho de la tabla se presenta información sobre el número de presidentes cubiertos por cada configuración y la proporción de estos casos en los que el Ejecutivo logró el control de la agenda parlamentaria. La última columna de la tabla identifica aquellas situaciones en las que los presidentes consistentemente han controlado la agenda

Tabla 2. Tabla de verdad

\begin{tabular}{lcccccccc}
\hline Código & $\begin{array}{c}\text { Potencia } \\
\text { legislativa }\end{array}$ & $\begin{array}{c}\text { Mayoría } \\
\text { parlamentaria }\end{array}$ & $\begin{array}{c}\text { Fragmentación } \\
\text { partidaria }\end{array}$ & $\begin{array}{c}\text { Distancia } \\
\text { ideológica }\end{array}$ & $\begin{array}{c}\text { (Con control } \\
\text { de la agenda) }\end{array}$ & Proporción & $\begin{array}{c}\text { iControl de } \\
\text { la agenda? }\end{array}$ \\
\hline 1111 & S & S & S & S & 2 & 2 & 1,00 & V \\
1101 & S & S & N & S & 1 & 1 & 1,00 & V \\
0101 & N & S & N & S & 1 & 1 & 1,00 & V \\
1001 & S & N & N & S & 2 & 2 & 1,00 & V \\
1011 & S & N & S & S & 4 & 3 & 0,75 & C \\
0100 & N & S & N & N & 7 & 4 & 0,57 & C \\
0000 & N & N & N & N & 6 & 1 & 0,17 & C \\
1000 & S & N & N & N & 1 & 0 & 0,00 & F \\
1110 & S & S & S & N & 1 & 0 & 0,00 & F \\
0111 & N & S & S & S & 1 & 0 & 0,00 & F \\
0001 & N & N & N & S & 2 & 0 & 0,00 & F \\
0010 & N & N & S & N & 2 & 0 & 0,00 & F \\
0011 & N & N & S & S & 0 & - & & R \\
0110 & N & S & S & N & 0 & - & & R \\
1010 & S & N & S & N & 0 & - & & R \\
1100 & S & S & N & N & 0 & - & & R \\
\hline
\end{tabular}

Notas: $\mathrm{N}$ indica la ausencia de un atributo discreto y $\mathrm{S}$ su presencia; $n$ corresponde al número de presidentes en cada grupo. La columna Proporción reporta el índice de consistencia para cada configuración. V indica una configuración verdadera (consistente con el resultado); C contradictoria (inconsistente); F falsa (consistente con la negación del resultado); R configuración residual. 
(configuraciones "verdaderas"), aquellas en que todos los presidentes han tenido una participación minoritaria en la producción legislativa ("falsas") y aquellas con resultados contradictorios. En este último grupo se distinguen dos situaciones en las que el control de la agenda es el resultado más probable y una tercera en que resulta improbable.

La Tabla 2 permite identificar así tres tipos de contexto institucional. Una primera región de la tabla, representada por las cuatro configuraciones listadas en el panel superior, refleja contextos en que el Ejecutivo tiene prácticamente garantizado el control de la agenda parlamentaria. Se utilizan en este texto calificativos como "prácticamente" o "virtualmente" para destacar que el análisis de estas configuraciones "verdaderas" no conlleva un argumento determinista. La región siguiente refleja situaciones en las que el control de la agenda es posible pero no está asegurado. De particular interés resultan las configuraciones 1011 y 0100, en que la mayoría de las administraciones han controlado la agenda. En la tercera región de la tabla, ningún presidente ha logrado controlar la agenda parlamentaria. Como complemento, el panel inferior de la tabla lista aquellas configuraciones lógicamente posibles para las que no tenemos ejemplos históricos entre los casos analizados (técnicamente denominadas residuos o remanentes de la tabla de verdad).

\section{ANÁLISIS COMPLEMENTARIO}

En esta sección extendemos el análisis previo en tres direcciones. En primer lugar, analizamos las configuraciones residuales (o contrafácticos) de la tabla de verdad para identificar posibles alternativas en el proceso de minimización lógica. En segundo lugar, analizamos los contextos institucionales en que el control parece inviable y aquellos en los que resulta apenas posible, para identificar las condiciones que impiden y favorecen este resultado. El análisis de estas dos regiones complementarias nos permite detectar las condiciones en donde el control presidencial de la agenda es el resultado más probable, aunque no esté asegurado. Estas conclusiones son verificadas por medio de un análisis estadístico de los niveles de participación presidencial en la producción legislativa, que muestra coherencia entre las conclusiones de las perspectivas cualitativa y cuantitativa.

\section{Configuraciones residuales}

El panel inferior de la Tabla 2 muestra cuatro situaciones para las que no tenemos ejemplos históricos en nuestra muestra de administraciones. Esto impone una reflexión sobre contrafácticos (Ragin y Sonnett, 2005). Si pudiésemos observar casos en estos contextos, ¿tendían estos presidentes garantizado el control de la agenda? Y, en tal caso, ¿conduciría el proceso de minimización lógica un conjunto más parsimonioso de conclusiones generales (implicantes primarios)? Las respuestas a estas dos preguntas son negativas. La primera configuración residual (0011) representa una situación en la que el Ejecutivo carece de potencia institucional y de mayorías parlamentarias, un contexto altamente adverso. Sabemos de una única administración (Wasmosy) que logró controlar la agenda bajo estas circunstancias, lo que sugiere que el control no resulta 
imposible, pero sí improbable. En las otras tres configuraciones $(0110,1010,1100)$ no existe una distancia ideológica clara entre los partidos en el Congreso, por lo que existen pocos incentivos para que el Ejecutivo busque controlar consistentemente la producción legislativa. Nuevamente, la Tabla 1 muestra que en esta situación el control de la agenda es posible, pero dista de ser un patrón predominante.

Incluso si estas cuatro configuraciones residuales fueran tratadas como tentativamente "verdaderas" (suficientes) al solo efecto de extender el proceso de minimización lógica, las conclusiones generales no se verían modificadas. La razón para este resultado puede apreciarse intuitivamente en la Figura 3. Los cuatro recuadros blancos, que representan las configuraciones residuales, no pueden anexarse a las configuraciones verdaderas, de manera de completar recuadros más amplios. Por ejemplo, si suponemos que la configuración 1100 es verdadera, se conforma un espacio en el cuadrante inferior derecho del gráfico que contiene a los presidentes con potencia institucional y mayoría parlamentaria (Bachelet, Lula y Torrijos). Sin embargo, este supuesto simplificador no permitiría concluir que la combinación de potencia institucional y mayorías es suficiente para garantizar el control de la agenda, porque el cuadrante incluye un caso (Uribe II) que refuta esta conclusión. Por lo tanto, la solución parsimoniosa del proceso de minimización, que incluye los residuos, es equivalente a la solución compleja, que los excluye.

\section{Condiciones que hacen el control inviable, viable y probable}

En esta sección realizamos tres análisis complementarios para detectar las condiciones que hacen del control de la agenda un resultado inviable, un resultado posible (aunque quizás improbable), y un resultado probable (aunque no garantizado). Para abordar la primera cuestión analizamos las configuraciones falsas. Es decir, aquellas en las que ningún presidente logró el control mayoritario de la agenda. ¿Qué tienen estas configuraciones en común? La Tabla 2 muestra que las cinco configuraciones falsas $(1000,1110,0111,0001$, y 0010) carecen de cualquier condición compartida. En dos de estas situaciones el presidente tenía potencia institucional, en otras dos el presidente contaba con mayorías parlamentarias, en dos situaciones el sistema de partidos no estaba fragmentado, y en dos instancias existía distancia ideológica clara entre los partidos. Por su estructura, estas cinco modalidades no pueden ser simplificadas mediante un proceso de minimización lógica, a menos que asumamos que algunas configuraciones residuales (para las que no hemos observado casos) hubiesen impedido el control presidencial de la agenda parlamentaria. Sin embargo, la reflexión de la sección anterior respalda una interpretación más cautelosa, ya que algunos episodios de control presidencial hubiesen sido posibles bajo estas configuraciones. De este modo, el análisis de las configuraciones falsas identifica situaciones históricas particulares a partir de ellas no es posible extraer una lección general.

Si aceptamos como conclusión tentativa que -bajo las configuraciones residuales- el control de la agenda no hubiese estado garantizado pero tampoco hubiese sido imposible, se pueden identificar (Tabla 2) tres tipos de situación en las que el control presidencial de la agenda parlamentaria resultaría viable: (1) las configuraciones verdaderas, en las 
que el resultado parece asegurado; (2) las configuraciones contradictorias, en las que es apenas posible; y (3) las configuraciones residuales, en las que suponemos, basados en la evidencia conocida, que el control presidencial sería viable en ciertas circunstancias.

El análisis de estas once configuraciones resulta en una solución parsimoniosa que remite a tres configuraciones genéricas en donde el control de la agenda sería un resultado viable:

[4] distancia*potencia

[5] mayoría* fragmentación

[6] distancia* $\sim$ potencia $*$ fragmentación

El presidente tiene la posibilidad de dominar la agenda legislativa cuando existe distancia ideológica entre los partidos y el presidente cuenta con poderes institucionales, cuando el presidente cuenta con una mayoría parlamentaria y el sistema de partidos no está fragmentado, y cuando el sistema de partidos no está fragmentado, aunque no exista una distinción ideológica clara y aunque el presidente no tenga poderes institucionales sólidos.

La primera solución es la más intuitiva y la de mayor capacidad explicativa. Cubre ocho de los catorce casos de control de la agenda (el 57\%), ubicados en un largo rectángulo vertical situado en el lado derecho de la Figura 3: Moscoso, Pastrana, Frei, Lagos, Aylwin, Bachelet, Lula y Torrijos. El control de la agenda en este grupo es altamente probable (el índice de consistencia es de 0,88 ), pero el caso de la administración Noboa muestra que el este resultado no está garantizado.

La segunda solución cubre seis de los catorce casos (43\%) ubicados en la parte inferior de la Figura 3: González Macchi, Sanguinetti, Reina, Sánchez de Lozada, Batlle y Torrijos. Dos terceras partes de los presidentes en este grupo lograron el control de la agenda (el índice de consistencia es de 0,67), pero los casos de Kirchner, Menem II y Zedillo prueban que el resultado no estaba asegurado.

La tercera solución es la menos intuitiva y la de menor capacidad explicativa. Poco más de un tercio de los casos de interés son cubiertos por esta explicación (González Macchi, Reina, Sánchez de Lozada, Sanguinetti y Wasmosy), pero solamente un caso (Wasmosy) no está cubierto por las explicaciones previas. El índice de consistencia en este grupo es distintivamente bajo $(0,38)$, lo que muestra que apenas una minoría de los presidentes en esta situación consigue dominar la agenda. La debilidad de esta explicación puede comprenderse al observar la Tabla 2. Dos configuraciones en la tabla son cubiertas por este implicante primario (0100 y 0000), pero solo la segunda configuración requiere de este implicante para ser explicada (el tipo 0100 está ya cubierto por la combinación mayoría* $\sim$ fragmentación). Solamente un presidente de los seis que experimentaron esta situación logró controlar la agenda parlamentaria, lo que confirma que el resultado de interés bajo estas circunstancias es posible, pero altamente improbable.

La principal conclusión de este análisis es que los implicantes primarios [4] y [5] reflejan condiciones institucionales que hacen el control presidencial de la agenda no sólo 
posible sino también probable; en tanto el implicante primario [6] captura un contexto institucional en el que este resultado es viable pero muy difícil de lograr cuando las mayorías parlamentarias son adversas.

La Tabla 3 verifica la lógica de esta conclusión, demostrando que los implicantes primarios que resumen las configuraciones verdaderas son un subconjunto de los implicantes primarios que resumen las configuraciones en las que el resultado es probable. La Tabla 3 utiliza los rótulos de control "probable" para destacar situaciones en las que el índice de consistencia es mayor a 0,5 y de control "virtualmente asegurado" para destacar situaciones en que el índice alcanza valores de 1,0 (aunque sin asumir una conclusión determinista). Cuando un presidente tiene potencia institucional y distancia ideológica de sus adversarios en el Congreso, es probable que logre el control de la agenda parlamentaria. Si este presidente se encuentra además beneficiado por una mayoría parlamentaria o por la ausencia de fragmentación en el Parlamento, el control de la agenda parece asegurado. De la misma forma, un presidente respaldado por una mayoría parlamentaria en un contexto de baja fragmentación probablemente logre el control de la agenda, pero si a este marco se agrega una justificación ideológica, el control de la agenda parece un resultado seguro.

Tabla 3. Condiciones que hacen el control probable y (virtualmente) seguro

\begin{tabular}{lc}
\hline \multicolumn{1}{c}{ Control probable } & \multicolumn{1}{c}{ Control asegurado } \\
\hline [4] distancia* potencia & {$[1]$ distancia* ${ }^{*}$ potencia*mayoría } \\
& {$[2]$ distancia* ${ }^{*}$ potencia* $\sim$ fragmentación } \\
[5] mayoría* $\sim$ fragmentación & {$[3]$ distancia*mayoría* $\sim$ fragmentación } \\
\hline
\end{tabular}

Nota: Control probable refiere a configuraciones con valores de consistencia mayores a 0,5 en la Tabla 2. Control virtualmente asegurado refiere a configuraciones con valores iguales a 1,0.

\section{Análisis de varianza}

¿Es posible que las conclusiones del análisis se vean distorsionadas por el tratamiento dicotómico de la variable dependiente? Como muestra la Figura 1, la participación legislativa de los gobiernos de Mireya Moscoso y de Ernesto Zedillo fue muy similar, pero la distinción binaria impone que estos dos casos sean clasificados en grupos distintos. Esto alerta sobre posibles sesgos de inferencia derivados de la estrategia de medición del control de la agenda parlamentaria.

A causa de que la tasa de participación ejecutiva sobre la producción legislativa es una variable continua, es posible analizar su varianza para establecer si los presidentes con mayores niveles de participación son los mismos que fueron identificados por el análisis cualitativo comparativo. Para ello creamos una variable nominal con 16 grupos, correspondientes a las configuraciones causales de la Tabla 2. Para las cuatro categorías residuales no hay casos observados, por lo que solamente se puede comparar el grado de participación legislativa de presidentes en 12 grupos. El análisis de varianza (anova) 
de un factor establece que esta clasificación explica más del $55 \%$ de la varianza de la variable dependiente continua $(\mathrm{F}=2,04, \mathrm{p}<0,1$, d.f. $=11)$.

La Tabla 4 reporta la media de participación para cada grupo, junto con una prueba de significación para detectar si estos valores son mayores al promedio de los presidentes con peor rendimiento (26\%). La prueba para cada grupo refleja el nivel de significación de un test t para los parámetros de una regresión tal que $y_{\mathrm{k}}=b_{0}+\sum b_{\mathrm{k}} d_{\mathrm{k}}$ en donde $y_{\mathrm{k}}$ es el nivel de participación en la producción parlamentaria para el k-gésimo tipo de administración, $b_{0}$ es la media de participación para el grupo con peor rendimiento (0010), tomado como categoría de referencia, $d_{\mathrm{k}}$ es un indicador dicotómico que identifica cada uno de los restantes 11 grupos, y $b_{\mathrm{k}}$ es el coeficiente para cada indicador.

Los resultados estadísticos permiten distinguir dos tipos de situaciones institucionales: unas con valores de participación estadísticamente mayores a las del grupo que representa el umbral inferior para la muestra de presidentes observados, y otras con valores no significativos, es decir, indistinguibles de la categoría de referencia. Para evaluar estos resultados la Tabla 4 presenta además el índice de consistencia (la proporción de presidentes que lograron el control de la agenda parlamentaria). Las categorías con valores significativos son coincidentes con las configuraciones verdaderas o con

Tabla 4. Participación en la producción legislativa (modelo saturado)

\begin{tabular}{|c|c|c|c|c|c|}
\hline Código & $\begin{array}{cc}\text { Potencia } & \text { Mayoría } \\
\text { legislativa } & \text { parlamentaria }\end{array}$ & $\begin{array}{cc}\text { Fragmentación } & \text { Distancia } \\
\text { partidaria } & \text { ideológica }\end{array}$ & N Proporción & $\begin{array}{l}\text { ¿Control de } \\
\text { la agenda? }\end{array}$ & $\begin{array}{c}\text { Media } \\
\text { de } \\
\text { participación }\end{array}$ \\
\hline
\end{tabular}

\begin{tabular}{lllllllll}
\hline 1111 & $\mathrm{~S}$ & $\mathrm{~S}$ & $\mathrm{~S}$ & $\mathrm{~S}$ & 2 & 1,00 & $\mathrm{~V}$ & $80,9^{* *}$ \\
1101 & $\mathrm{~S}$ & $\mathrm{~S}$ & $\mathrm{~N}$ & $\mathrm{~S}$ & 1 & 1,00 & $\mathrm{~V}$ & $64,2^{*}$ \\
0101 & $\mathrm{~N}$ & $\mathrm{~S}$ & $\mathrm{~N}$ & $\mathrm{~S}$ & 1 & 1,00 & $\mathrm{~V}$ & $65,7^{* *}$ \\
1001 & $\mathrm{~S}$ & $\mathrm{~N}$ & $\mathrm{~N}$ & $\mathrm{~S}$ & 2 & 1,00 & $\mathrm{~V}$ & $56,4^{*}$ \\
1011 & $\mathrm{~S}$ & $\mathrm{~N}$ & $\mathrm{~S}$ & $\mathrm{~S}$ & 4 & 0,75 & $\mathrm{C}$ & $54,2^{* *}$ \\
0100 & $\mathrm{~N}$ & $\mathrm{~S}$ & $\mathrm{~N}$ & $\mathrm{~N}$ & 7 & 0,57 & $\mathrm{C}$ & $59,4^{* *}$ \\
0000 & $\mathrm{~N}$ & $\mathrm{~N}$ & $\mathrm{~N}$ & $\mathrm{~N}$ & 6 & 0,17 & $\mathrm{C}$ & 46,3 \\
1000 & $\mathrm{~S}$ & $\mathrm{~N}$ & $\mathrm{~N}$ & $\mathrm{~N}$ & 1 & 0,00 & $\mathrm{~F}$ & 44,5 \\
1110 & $\mathrm{~S}$ & $\mathrm{~S}$ & $\mathrm{~S}$ & $\mathrm{~N}$ & 1 & 0,00 & $\mathrm{~F}$ & 40,0 \\
0111 & $\mathrm{~N}$ & $\mathrm{~S}$ & $\mathrm{~S}$ & $\mathrm{~S}$ & 1 & 0,00 & $\mathrm{~F}$ & 39,4 \\
0001 & $\mathrm{~N}$ & $\mathrm{~N}$ & $\mathrm{~N}$ & $\mathrm{~S}$ & 2 & 0,00 & $\mathrm{~F}$ & 31,9 \\
0010 & $\mathrm{~N}$ & $\mathrm{~N}$ & $\mathrm{~S}$ & $\mathrm{~N}$ & 2 & 0,00 & $\mathrm{~F}$ & $26,0 \#$ \\
0011 & $\mathrm{~N}$ & $\mathrm{~N}$ & $\mathrm{~S}$ & $\mathrm{~S}$ & 0 & & $\mathrm{R}$ & s.d. \\
0110 & $\mathrm{~N}$ & $\mathrm{~S}$ & $\mathrm{~S}$ & $\mathrm{~N}$ & 0 & & $\mathrm{R}$ & s.d. \\
1010 & $\mathrm{~S}$ & $\mathrm{~N}$ & $\mathrm{~S}$ & $\mathrm{~N}$ & 0 & & $\mathrm{R}$ & s.d. \\
1100 & $\mathrm{~S}$ & $\mathrm{~S}$ & $\mathrm{~N}$ & $\mathrm{~N}$ & 0 & & $\mathrm{R}$ & s.d. \\
\hline
\end{tabular}

Las pruebas para cada grupo reflejan si la media es estadísticamente mayor al umbral mínimo de participación $(26 \%)$. ${ }^{*} \mathrm{p}<0,1{ }^{* *} \mathrm{p}<0,05$ \# Categoría de referencia $(\mathrm{p}<0,05) \cdot \mathrm{R}^{2}=0,555(\mathrm{~N}=30$, d.f. $=11)$. 
las configuraciones contradictorias en que el control de la agenda es un resultado probable, en tanto que las categorías con valores no significativos son coincidentes con las configuraciones falsas y con la única configuración contradictoria con una media inferior al 50\% (0000). Esta última configuración es la que queda fuera de nuestras conclusiones cuando eliminamos el implicante primario [6]. Los análisis cualitativo y cuantitativo producen así resultados coherentes, tanto cuando se mide el control de la agenda legislativa de manera dicotómica, como de manera continua. Las configuraciones institucionales que nos permiten explicar situaciones de "control probable" permiten también explicar aquellas situaciones en que la media de participación, tratada como una variable continua, supera el $50 \%$.

\section{CONCLUSIONES}

Los resultados de este artículo sugieren tres contribuciones metodológicas y dos contribuciones sustantivas al análisis del poder presidencial en América Latina. En primer lugar, este trabajo ha introducido un modo original de conceptualizar el control presidencial de la agenda parlamentaria, definido como una participación mayoritaria del Poder Ejecutivo en la producción legislativa. Este enfoque permite distinguir la cuestión del dominio presidencial sobre la agenda de otras cuestiones relacionadas, como la capacidad presidencial de obtener aprobación parlamentaria para sus proyectos (Saiegh, 2011; Steger, 1997). En segundo lugar, el estudio ha demostrado la utilidad del análisis cualitativo comparativo (QCA) para interpretar mecanismos causales en los que varias condiciones institucionales interactúan para definir la formación de políticas públicas (Pérez Liñán y Rodríguez Raga, 2009). Como complemento de este enfoque, una tercera contribución radica en el uso de un modelo estadístico saturado para verificar las conclusiones del análisis cualitativo, encontrando una perspectiva común para integrar los modelos de regresión y el enfoque de QCA (Seawright, 2005).

Las dos conclusiones sustantivas refieren a los contextos que explican la influencia del Ejecutivo y sus consecuencias para la vida democrática. ¿Bajo qué condiciones los presidentes logran dominar la producción legislativa? La respuesta a esta cuestión implica reconocer que ningún factor causal puede garantizar aisladamente el éxito del presidente frente al Parlamento. Nuestro análisis sugiere que la combinación de distancia ideológica y potencia institucional es la principal combinación histórica que torna probable el control presidencial de la agenda. Cuando estas condiciones se ven reforzadas por la presencia de una mayoría parlamentaria o por un sistema partidario poco fragmentado, parece casi seguro que el Ejecutivo domine la agenda. De manera alternativa, la presencia de una mayoría parlamentaria fiel al Ejecutivo en un sistema partidario poco fragmentado también facilita el control de la agenda, y estos mecanismos se refuerzan en contextos de clara distancia ideológica entre los partidos.

El análisis tiende a destacar así que la combinación de un diseño institucional favorable y una división del Congreso en bloques ideológicos diferenciados favorece al Poder Ejecutivo. Pero estos factores no operan de manera aislada. Los resultados confirman la presencia de efectos condicionales o de causalidad coyuntural (Ragin 1987), ya que solo 
la articulación de ciertos factores en configuraciones históricas garantiza el resultado, y la presencia de equifinalidad o causalidad múltiple (George y Bennett, 2005), porque existen vías alternativas para lograr una participación mayoritaria en la producción legislativa.

La conclusión final retorna a las primeras páginas de este artículo. ¿Son los contextos que promueven el control presidencial de la agenda fuentes de eficiencia legislativa o causas del gobierno arbitrario por parte del Ejecutivo? ¿Generan estos mecanismos marcos institucionales favorables o adversos al proceso de democratización? No es posible ofrecer respuestas definitivas en este artículo, pero un ejercicio comparativo permite orientar la reflexión sobre este tema. Los países de nuestro estudio con un patrón consistente de control presidencial de la agenda legislativa son Brasil, Chile, Panamá, Paraguay y Uruguay. ${ }^{17}$ Con la posible excepción de Paraguay, estos ejemplos no coinciden con casos endémicos de democracia delegativa ( $\mathrm{O}^{\prime}$ Donnell, 1994) ni con las sociedades con mayor déficit democrático en nuestra muestra. Entre los tres países con una tradición democrática más sólida, dos se encuentran en este bloque (Chile y Uruguay) y el tercero (Costa Rica) no presenta ninguna instancia de control presidencial de la agenda. El análisis de unas pocas administraciones por caso no nos permite formular amplias generalizaciones históricas sobre el funcionamiento institucional del Ejecutivo en cada país. Pero estos resultados muestran que si bien la relación entre control de la agenda y funcionamiento institucional es compleja, no existe una tensión intrínseca entre una mayor eficiencia legislativa y una mayor democratización.

\section{REFERENCIAS}

Ajenjo Fresno, Natalia. 2004. "El papel y función de los Parlamentos centroamericanos: cuatro casos comparados". América Latina Hoy 38: 125-139.

Alcántara Sáez, Manuel, Mercedes García Montero y Francisco S. López. 2005. Funciones, procedimientos y escenarios: un análisis del Poder Legislativo en América Latina. Salamanca: Ediciones Universidad de Salamanca.

Alcántara Sáez, Manuel y Mercedes García Montero. 2008. "Institutions and Politicians: An Analysis of the Factors that Determine Presidential Legislative Success". Working paper $N^{\circ} 348$, The Hellen Kellogg Institute for International Studies, Universidad de Notre Dame.

Alemán, Eduardo y George Tsebelis. 2005. “The Origins of Presidential Conditional Agenda-Setting Power in Latin America". Latin American Research Review 40 (2): 3-26.

Alemán, Eduardo y Patricio Navia. 2010. “Institutions and the Legislative Success of 'Strong' Presidents: An Analysis of Government Bills in Chile". Journal of Legislative Studies 15 (4): 401-419.

Alemán, Eduardo y Ernesto Calvo. 2010. "Unified Government, Bill Approval, and the Legislative Weight of the President". Comparative Political Studies 43 (4): 511-534.

17 Si bien nuestro análisis incluye una sola administración de Brasil como resultado de la ausencia de datos de polarización ideológica anteriores a 2003, es importante destacar que la tasa de participación de otros presidentes brasileños ha sido igualmente elevada: en la primera administración Cardoso (1995-1999), la tasa fue del 85,4\%; en la segunda administración Cardoso (1999-2003), ésta se mantuvo en 81,5\%. Como contraste, nótese que la tasa de participación de la primera administración Menem en Argentina (1989-1995) fue del 46, $6 \%$, muy similar a las observadas en su segundo mandato y en la administración Kirchner. 
Altman, David. 2001.“Crisis de gobernabilidad democrática: orígenes y mapa de lectura”. Revista Instituciones y Desarrollo 8 y 9: 385- 410. Barcelona: Institut Internacional de Governabilitat de Catalunya.

Amorim Neto, Octavio. 1998. "Cabinet Formation in Presidential Regimes: An Analysis of 10 Latin American Countries", paper prepared for delivery at the 1998 meeting of the Latin American Studies Association, Chicago, Illinois, September.

Amorim Neto, Octavio y Fabiano Santos. 2003. “O segredo Ineficiente Revisto: O que propõem e o que Aprovam os Deputados Brasileiros". Dados Revista de Ciências Sociais 46 (4): 661-698.

Amorim Neto, Octavio y Eric Magar. 2000. "Veto Bargaining and Coalition Formation: A Theory of Presidential Policymaking with Application to Venezuela", paper prepared for delivery at the 2000 meeting of the Latin American Studies Association.

Baldez, Lisa y John M.Carey. 1999. "Presidential Agenda Control and Spending Policy: Lessons from General Pinochet's Constitution". American Journal of Political Science 43 (1): 29-55.

Calvo, Ernesto. 2004. "The Responsive Legislature: Public Opinion and Law Making in a Highly Disciplined Legislature". Trabajo presentado en UNC-Duke Political Economy, Workshop.

Calvo, Ernesto. 2008. "Nuevos estudios sobre el Poder Legislativo". En Investigaciones actuales sobre Estado, Instituciones Políticas y Sociedad, 17-38. Buenos Aires: Secretaría de Gabinete y Gestión Pública.

Cárdenas, Mauricio, Roberto Junguito y Mónica Pachón. 2008. “Political Institutions and Policy Outcomes in Colombia: The Effects of the 1991 Constitution". En Policy Making in Latin America How Politics Shapes Policies, editado por Ernesto Stein y Mariano Tommasi. Washington: BID.

Carey, John y Matthew S. Shugart. 1998. Executive Decree Authority. Cambridge: Cambridge University Press.

Casar, María Amparo. 2002. "Executive-Legislative Relations: The Case of Mexico (1946-1997)". En Legislative Politics in Latin America, editado por Scott Morgenstern y Benito Nacif. Cambridge: Cambridge University Press.

Carroll, Royce y Mónica Pachón. 2012. “The Legislative Agenda in Presidential Democracies". Documento de trabajo. Rice University y Universidad de los Andes.

Ragin, Charles C.y John Sonnett. 2005. "Between Complexity and Parsimony: Limited Diversity, Counterfactual Cases, and Comparative Analysis". Em Vergleichen in der Politikwissenschaft, editado por Sabine Kropp y Michael Minkenberg. Reimpresso 2008. “Limited Diversity and Counterfactual Cases". En Redesigning Social Inquiry, editado por Charles C. Ragin.

Chasquetti, Daniel. 2001. “Democracia, multipartidismo y coaliciones en América Latina: evaluando la difícil combinación". En Tipos de presidencialismo y coaliciones políticas en América Latina, editado por Jorge Lanzaro. Buenos Aires: CLACSO.

Chasquetti, Daniel. 2008. Democracia, presidencialismo y partidos políticos en América Latina: Evaluando la difícil combinación. Montevideo: Cauce.

Colomer, Josep M. y Gabriel Negretto. 2005. "Can Presidentialism Work like Parliamentarism?". Government and Opposition 40 (1): 60-89.

Cox, Gary. 2006. "The Organization of Democratic Legislatures". En The Oxford Handbook of Political Economy, editado por Barry Weingast y Donald Wittman. Oxford: Oxford University Press.

Cox, Gary W. y Matthew McCubbins. 1999. "The Institutional Determinants of Economic Policy Outcomes". Presentado en la Annual Conference of the International Society for New Institutional Economics, Washington.

Cox, Gary W. y Scott Morgenstern.2001. "Latin America's Reactive Assemblies and Proactive Presidents". Comparative Politics 33 (2): 171-190.

Cronqvist, Lasse. 2005. "Introduction to Multi-Value Qualitative Comparative Analysis. MVQCA)". Marburg: Institute of Political Science, Philipps-University.

Ferreira Rubio, Delia y Mateo Goretti. 1996. "Cuando el presidente gobierna solo. Menem y los decretos de necesidad y urgencia hasta la reforma constitucional (julio 1989-agosto 1994)". Desarrollo Económico 36 (141): 443-474.

Figueiredo, Argelina y Fernando Limongi. 1999. Executivo e Legislativo na Nova Ordem Constitucional. Rio de Janeiro: Editora FGV.

Figueiredo, Argelina; Dense Lopes Salles y Marcelo Martins Vieira. 2011. “Determinantes políticos e institucionales del Éxito Legislativo del Ejecutivo en América Latina". En Algo más que presidentes. 
El papel del Poder Legislativo en América Latina, editado por Manuel Alcántara Sáez y Mercedes García Montero. Zaragoza: Fundación Manuel Giménez Abad.

García Montero, Mercedes. 2007. "La actividad legislativa en América Latina: sobre el papel reactivo y proactivo de Presidentes y Parlamentos". Lateinamerika Analysen 17 (2): 3-24.

García Montero, Mercedes. 2009. Presidentes y Parlamentos ¿quién controla la actividad legislativa en América Latina?. Madrid: Centro de Investigaciones Sociológicas.

George, Alexander L., y Andrew Bennett. 2005. Case Studies and Theory Development in the Social Sciences. Cambridge, Mass.: MIT Press.

Huneeus, Carlos y Fabiola Berríos. 2005. "El Congreso en el presidencialismo. El caso de Chile". Revista SAAP 2 (2): 345-391.

Hurtado González, Javier. 2002. "Relaciones Presidencia-Congreso en el proceso legislativo del México de nuestros días". En Relaciones entre Gobierno y Congreso, editado por Cecilia Mora-Donato. Memoria del VII Congreso Iberoamericano de Derecho Constitucional. México: Universidad Nacional Autónoma de México.

Jones, Mark P., Sebastián Saiegh, Pablo T. Spiller y Mariano Tommasi. 2000. "Políticos profesionales legisladores 'amateurs': el Congreso argentino en el siglo XX". Artículo preparado para presentar en la Conferencia Anual de la Sociedad Internacional de la Nueva Economía Institucional del 22 al 24 de septiembre de 2000, Tübingen.

Krehbiel, Keith. 1998. Pivotal Politics: A Theory of U.S. Lawmaking. Chicago: The University of Chicago Press. Laakso, Markku y Rein Taagepera. 1979. "Effective number of parties: A measure with application to Western Europe". Comparative Political Studies 12 (1): 3-27.

Lanzaro, Jorge. 2000. Estudio de la producción legislativa 1985-2000. Montevideo: Instituto de Ciencia Política. Lieberson, Stanley. 1991. "Small N's and Big Conclusions: An Examination of the Reasoning in Comparative Studies Based on a Small Number of Cases". Social Forces 70 (2): 307-320.

Linz, Juan José. 1990. "The Perils of Presidentialism". Journal of Democracy 1 (1): 51-69.

Magar, Erik y Juan Andrés Moraes. 2007. "Coalición y resultados: aprobación y duración del trámite parlamentario en Uruguay (1985-2000)". Revista Uruguaya de Ciencia Política 17 (1): 39-70.

Mainwaring, Scott. 1993. "Presidentialism, Multipartism, and Democracy: The Difficult Combination". Comparative Political Studies 26 (2): 198-228.

Mainwaring, Scott y Mathew S. Shugart (edits.). 1997. Presidentialism and Democracy in Latin America. Cambridge: Cambridge University Press. En español Scott Mainwaring y Matthew Soberg Shugart. (comps.). 2002. Presidencialismo y democracia en América Latina. Buenos Aires: Paidós.

Mainwaring, Scott y Timothy Scully (edits.). 1995. Building Democratic Institutions: Party Systems in Latin America. Stanford: Stanford University Press.

Mejía Acosta, Andrés. 2000. "Weak Coalitions and Policy Making in the Ecuatorian Congress. 1979-1996". Paper prepared for meeting of the Latin American Studies Association, Miami 16, 17 march.

Metcalf, Lee Kendall. 2000. "Measuring Presidential Power". Comparative Political Studies 33 (5): 660-685. Mill, John Stuart. 1843/1967. A System of Logic: Ratiocinative and Inductive. Toronto: University of Toronto Press. Molas, Isidro e Ismael Pitarch. 1987. Las Cortes Generales en el sistema parlamentario de Gobierno. Madrid: Tecnos.

Molinas, José, Aníbal Pérez Liñán y Sebastián Saiegh. 2004. “Political Institutions, Policymaking Proceses, and Policy Outcomes in Paraguay, 1954-2003". Revista de Ciencia Política 24 (2): 67-93.

Molinelli, Guillermo, Valeria Palanza y Gisela Sin. 1999. Congreso, Presidencia y Justicia en Argentina. Buenos Aires: Temas Grupo Editorial

Morgenstern, Scott y Benito Nacif. (eds.). 2002. Legislative Politics in Latin America. Nueva York: Cambridge University Press.

Mustapic, Ana María. 2002. "Oscilating Relations: President and Congress in Argentina". En Legislative Politics in Latin America, editado por Scott Morgenstern y Benito Nacif. Cambridge: Cambridge University Press.

Nacif, Benito. 2005. "The Fall of the Dominant Presidency: Lawmaking under Divided Government in Mexico". Paper prepared for presentation at the conference What Kind of Democracy Has Mexico? The Evolution of Presidentialism and Federalism, Center for U.S.-Mexican Studies, UCSD, March.

Nava Polina, María del Carmen y Jorge Yáñez López. 2003 “The Effects of Pluralism in the Legislative Activity: The Mexican Chamber of Deputies, 1917-2000". Prepared for delivery at The International 
Research Workshop, organized by the Center For New Institutional Social Sciences, Washington University in St. Louis, St. Louis, Missouri, USA.

Navarro, Karlos. 2004. ¿A quiénes representan y para quiénes legislan los diputados? Crisis de representatividad en la Asamblea Nacional. Managua: Foro Democrático.

Negretto, Gabriel. 2003. "Diseño constitucional y separación de poderes en América Latina". Revista Mexicana de Sociología 1: 41-76.

Negretto, Gabriel L. 2013. Making Constitutions. Presidents, Parties, and Institutional Choice in Latin America. Cambridge: Cambridge University Press.

Nino, Carlos. 1996. "Hyper-Presidentialism and Constitutional Reform". En Institutional Design in New Democracies, editado por Arend Lijphart y Carlos Waisman. Boulder: Westview.

O’Donnell, Guillermo. 1994. "Delegative Democracy". Journal of Democracy 5 (1): 55-69.

Oñate, Pablo y Francisco A. Ocaña. 1999. Análisis de datos electorales. Cuadernos Metodológicos, $n^{\circ} 27$. Madrid: CIS.

Payne, J. Mark, Daniel Zovatto, Fernando Carrillo Florez y Andrés Allamad Zavala. 2003. La política importa. Democracia y desarrollo en América Latina. Washington: BID.

Pérez Liñán, Aníbal. 2000. "¿Juicio político o golpe legislativo?: sobre las crisis institucionales en los años noventa". América latina hoy 26: 67-74.

Pérez-Liñán, Aníbal, and Juan Carlos Rodríguez-Raga. 2009. “Veto Players in Presidential Regimes: Institutional Variables and Policy Change". Revista de Ciencia Política 29 (3): 693-720.

PNUD. 2005. La democracia en América Latina: Hacia una democracia de ciudadanas y ciudadanos. Buenos Aires: Aguilar, Altea, Taurus, Alfaguara.

Ragin, Charles C. 1987. The Comparative Method. Berkeley: University of California Press.

Ragin, Charles C. 2000. Fuzzy-Set Social Science. Chicago: University of Chicago Press.

Ragin, Charles C. 2003. Recent Advances in Fuzzy-Set Methods and their Application to Policy Questions. Working paper posted at http://www.compasss.org/WP.htm.

Ragin, Charles C. 2006. "Set Relations in Social Research: Evaluating Their Consistency and Coverage". Political Analysis 14 (3): 291-310.

Ragin, Charles C. 2008. Redisigning Social Inquiry: Fuzzy Sets and Beyond. Chicago: University of Chicago Press. Ragin, Charles C., y John Sonnett. 2005. "Between Complexity and Parsimony: Limited Diversity, Counterfactual Cases, and Comparative Analysis". En Vergleichen in der Politikwissenschaft, editado por S. Kroppy M. Minkenberg. Wiesbaden: Verlagfür Sozialwissenschaften.

Ricci, Paolo. 2002. “Conexão eleitoral e estratégias legislativas: hipóteses e evidências para o caso brasileiro". Ponencia presentada en el $3^{\text {er }}$ Encuentro de la Asociación Brasileña de Ciencia Política Democracia y Justicia Social.

Saiegh, Sebastián. 2010. “Jugadores activos o apéndices del ejecutivo? una evaluación del papel de los legisladores latinoamericanos en la toma de decisiones". Política y gobierno 17 (1): 3-24.

Saiegh, Sebastián. 2011. Ruling by Statute: How Uncertainty and Vote Buying Shape Lawmaking. Cambridge: Cambridge University Press.

Samuels, David. 2002. "Progressive Ambition, Federalism, and Pork-Barreling in Brazil". En Legislative Politics in Latin America, editado por Scott Morgenstern y Benito Nacif. Cambridge: Cambridge University Press.

Samuels, David y Matthew S. Shugart.2003. "Presidentialism, Elections and Representation". Journal of Theoretical Politics15 (1): 33-60.

Sartori, Giovanni. 1999. Partidos y sistemas de partidos. Madrid: Alianza Editorial.

Schneider, Carsten Q. y Claudius Wagemann. 2006. "Reducing Complexity in Qualitative Comparative Analysis. QCA. Remote and Proximate Factors and the Consolidation of Democracy". European Journal of Political Research 45 (5): 751-786.

Schneider, Carsten Q. y Claudius Wagemann. 2012. Set-Theoretic Methods for the Social Sciences: A Guide to Qualitative Comparative Analysis. Cambridge: Cambridge University Press.

Seawright, Jason. 2005. "Qualitative Comparative Analysis vis-à-vis Regression". Studies in Comparative International Development 40 (1): 3-26.

Shugart, Matthew Soberg y John Carey. 1992. Presidents and Assemblies: Constitutional design and electoral dynamics. Cambridge: Cambridge University Press. 
Shugart, Matthew Soberg y Stephan Haggard.2001. "Institutions and Public Policy in Presidential Systems". En Presidents, Parliaments, and Policy, editado por Stephan Haggard y Mathew D. McCubbins. Nueva York: Cambridge University Press.

Siavelis, Peter M. 2002. "Exaggerated Presidentialism and Moderate Presidents: Executive-Legislative Relations in Chile". En Legislative Politics in Latin America, editado por Scott Morgenstern y Benito Nacif. Cambridge: Cambridge University Press.

Skocpol, Theda, y Margaret Somers. 1980. "The Uses of Comparative History in Macrosocial Inquiry". Comparative Studies in Society and History 22 (2): 174-197.

Steger, Wayne P. 1997. "Presidential Policy Initiation and the Politics of Agenda Control". Congress $\&$ the Presidency 24 (1): 17-36.

Stein, Ernesto, Mariano Tommasi, Koldo Echebarría, Eduardo Lora y Mark Payne. 2005. The Politics of Policies. Economic and Social Progress in Latin America 2006 REPORT. Washington: Inter-American Development Bank; David Rockefeller Center for Latin American Studies y Harvard University.

Taylor Robinson, Michele y Christopher Díaz. 1999. "Who Gets Legislation Passed in a Marginal Legislature and is the Label Marginal Legislature Still Appropriate? A Study of the Honduran Congress". Comparative Political Studies 32 (5): 589-625.

Weldon, Jeffrey A.2004. "The Spring 2004 Term of the Mexican Congreso". Mexican congressional REPORT series, CSIS.

Manoel Leonardo Santos es profesor del Departamento de Ciencia Política de la Universidade Federal de Minas Gerais. Director Adjunto del Centro de Estudios Legislativos de la UFMG. Investigador visitante del Instituto de Pesquisa Econômica Aplicada (IPEA-Brazil)). Master y Doctor en Ciencia Política en la Universidade Federal de Pernambuco (Brazil). Especialista en Poder Legislativo por PUC/Minas. Es el autor de Voto, Parlamento e Políticas Públicas (Editora de la Universidade Federal de Pernambuco, Brazil, 2009). E-mail: manoelsantos@fafich.ufmg.br

Aníbal Pérez Liñán es profesor de ciencia política e investigador del Centro de Estudios Latinoamericanos en la Universidad de Pittsburgh. Es autor de los libros Juicio político al presidente y nueva inestabilidad política en América Latina (Fondo de Cultura Económica, 2009; Cambridge UniversityPress, 2007) y Democracies and Dictatorships in Latin America: Emergence, Survival, and Fall (con Scott Mainwaring, Cambridge University Press, 2013), así como de numerosos artículos académicos. E-mail: asp27@pitt.edu

Mercedes García Montero es profesora en el área de Ciencia Política de la Universidad de Salamanca. Doctora en Ciencia Política y de la Administración, Master en Estudios Latinoamericanos y Licenciada en Sociología por la Universidad de Salamanca. Es investigadora del Instituto de Iberoamérica de la Universidad de Salamanca y ha publicado distintos trabajos sobre el poder legislativo en América Latina, entre los que destacan los libros Presidentes y Parlamentos ¿quién controla la actividad legislativa en América Latina? (CIS, 2010); Funciones, procedimientos y escenarios: un análisis del poder legislativo en América Latina (USAL, 2005), escrito junto a Manuel Alcántara y Francisco Sánchez López (publicación que recibió el premio de la Asociación Española de Ciencia Política y de la Administración AECPA en 2006). E-mail: mercegar@usal.es 